\title{
Quantifying examination distance in ophthalmic assessments
}

\author{
George Moussa $^{1} \cdot$ Priyanka Mandal $^{1} \cdot$ Namita Mathews $^{1} \cdot$ Rynn Lee $^{1}$
}

Received: 2 May 2020 / Revised: 13 May 2020 / Accepted: 13 May 2020 / Published online: 20 May 2020

(C) The Royal College of Ophthalmologists 2020

\section{To the Editor:}

In response to 'Controversies regarding mask usage in ophthalmic units in the United Kingdom during the COVID-19 pandemic' [1].

We read the above comment with interest. As of 2nd May 2020, COVID-19 has claimed 229,971 lives and there are over 3 million confirmed cases [2]. The World Health Organisation notes that transmission of the virus can occur via droplet spread within $1 \mathrm{~m}$. As Ophthalmologists, examination and treatment at close proximity is our daily practice. Ophthalmic clinical reviews are currently classified in the same category as other specialties with minimal levels of personal protective equipment (PPE) advised. These recommendations also apply to prolonged procedures, such as slit-lamp laser treatment [3].

Using a range of routinely employed ophthalmic equipment, we measured the nose-to-nose distance between the clinician and patient during these encounters. The measurements were repeated three times and a mean was taken (Table 1).

Figure 1 is illustrative of common ophthalmic on-call referrals. An 81-year-old gentleman was admitted out-ofhours with suspected orbital cellulitis secondary to facial cellulitis. This necessitated bedside review by the on-call Ophthalmology registrar using a portable slit-lamp and indirect ophthalmoscope at a distance of 13 and $71 \mathrm{~cm}$, respectively. The examination took several minutes to complete due to extensive oedema and patient discomfort. The patient also coughed at several points during the examination. This patient had not been deemed a COVID19 suspect, nor was he in a high-risk area. At the time of his assessment, guidelines recommended that only a surgical mask and slit-lamp guard for COVID-19 suspected or confirmed cases was required. Public Health England (PHE)

George Moussa

george.moussa@nhs.net

1 University Hospital Coventry and Warwickshire, Coventry, UK and the Royal College of Ophthalmology (RCOphth) now recommend the use of a fluid-resistant type IIR surgical mask, eye protection, disposable gloves, and plastic apron when assessing all patients, regardless of COVID-19 status. However, a recent survey of Ophthalmologist's reflected anxiety and a lack of confidence concerning COVID-19 exposure risk in an ophthalmic setting [4]. Consequently, standalone eye hospitals introduced PPE standards beyond recommendations from PHE and RCOphth, such as FFP3 respirators for all clinical staff in A\&E [4].

Ophthalmologists continue to provide emergency services during the COVID-19 outbreak. Bedside reviews lend to small distances, far less than $2 \mathrm{~m}$, perhaps more often than appreciated.

Table 1 Nose-to-nose measurement between clinician and patient.

\begin{tabular}{ll}
\hline Examination medium & Nose-to-nose measurement $(\mathrm{cm})$ \\
\hline Direct ophthalmoscope & -0.5 (overlap) \\
Portable slit Lamp & 13 \\
Slit-lamp examination & 27 \\
Indirect ophthalmolscope & 71 \\
\hline
\end{tabular}

Measurements dependent on anatomical variance between clinicians and patients, and the nature of the examination.

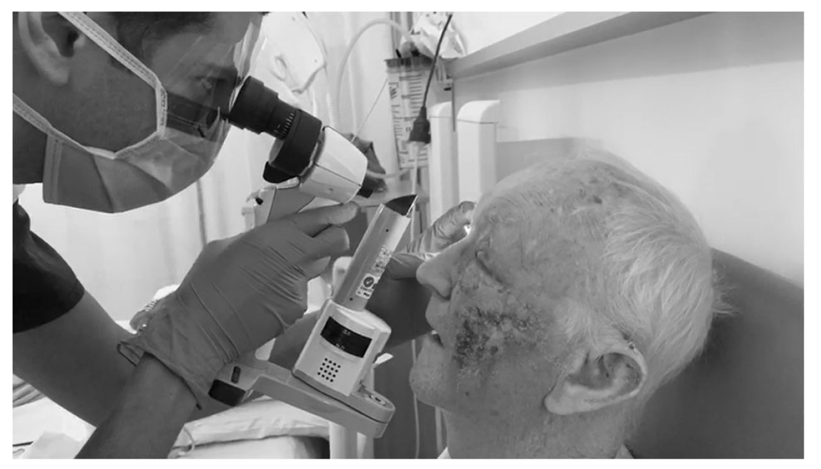

Fig. 1 Illustration of common on-call activity by Ophthalmologists. Measurements dependent on anatomical variance between clinicians and patients, and the nature of the examination. 


\section{Compliance with ethical standards}

Conflict of interest The authors declare that they have no conflict of interest.

Publisher's note Springer Nature remains neutral with regard to jurisdictional claims in published maps and institutional affiliations.

\section{References}

1. Naveed H, Scantling-Birch Y, Lee H, Nanavaty MA. Controversies regarding mask usage in ophthalmic units in the United Kingdom during the COVID-19 pandemic. Eye. 2020. http://www.nature. com/articles/s41433-020-0892-2.

2. Anon. World Health Organization, Coronavirus disease 2019 (COVID-19) Situation Report-103. https://www.who.int/docs/ default-source/coronaviruse/situation-reports/20200502-covid-19sitrep-103.pdf?sfvrsn $=$ d95e76d8_4.

3. Ophthalmologists RC of PPE requirements for ophthalmology. https://www.rcophth.ac.uk/wp-content/uploads/2020/04/ UPDATED-RCOphth-PPE-Principles-for-ophthalmic-staffprotection-090420.pdf.

4. Minocha A, Sim SY, Than J, Vakros G. Survey of ophthalmology practitioners in A\&E on current COVID-19 guidance at three Major UK Eye Hospitals. Eye. 2020. https://www.nature.com/articles/ s41433-020-0857-5. 